\title{
The safety and efficacy of front-firing green-light laser endoscopic en bloc photoselective vapo- enucleation of non-muscle-invasive bladder cancer
}

This article was published in the following Dove Press journal:

Therapeutics and Clinical Risk Management

II August 2017

Number of times this article has been viewed

\author{
Bo Cheng ${ }^{1,2}$ \\ Xiaofu Qiu' \\ Huanhui Li' \\ Guosheng Yang' \\ 'Department of Urology, Southern \\ Medical University affiliated \\ Guangdong Second Provincial General \\ Hospital, Southern Medical University, \\ Guangzhou, People's Republic of \\ China; ${ }^{2}$ Department of Urology, The \\ Affiliated Hospital of Southwest \\ Medical University, Luzhou, People's \\ Republic of China
}

Purpose: Laser therapy provides an alternative option for treating non-muscle-invasive bladder cancer (NMIBC). However, the clinical evidence for potassium-titanyl-phosphate (KTP) laser en bloc resection is still limited. Here, we investigated the efficacy and safety of the $120-\mathrm{W}$ front-firing KTP laser for the treatment of NMIBC.

Methods: A total of 64 patients with NMIBC treated with either a $120-\mathrm{W}$ front-firing KTPphotoselective vapo-enucleation of the bladder tumor (PVEBT, $n=34$ ) or transurethral resection of the bladder tumor (TURBT, n=30) were included. En bloc resection was applied to the patients in PVEBT group.

Results: There was no significant difference in rinsing time $(P=0.292)$, indwelling catheter $(P=0.080)$, pathologic type, and T stage $(P=0.870)$ between the two groups. Compared with the TURBT group, patients treated with PVEBT had a shorter hospitalization stay $(P=0.044)$, a shorter operation time $(P=0.008)$, and a lower muscle miss rate $(P=0.044)$. PVEBT is superior to TURBT in terms of the rate of 1 -year recurrence $(P=0.015)$ and tumor grade progression rate $(P=0.019)$.

Conclusion: The 120-W front-firing KTP laser en bloc enucleation technique is a safe and feasible procedure for treating patients with NMIBC. Further external validation in larger cohorts with a long follow-up period is warranted.

Keywords: bladder cancer, transurethral resection, en bloc, laser surgery, recurrence

\section{Introduction}

Bladder cancer is the second most common genitourinary malignancy, with an estimated 76,960 new cases and 16,390 new deaths in 2016 in the United States. ${ }^{1}$ Approximately $75 \%$ of cases have non-muscle-invasive bladder cancer (NMIBC) at diagnosis. ${ }^{2}$ Unfortunately, nearly half of the NMIBC patients have a high propensity to recurrence or progression to muscle-invasive bladder cancer, which is considered a highly lethal and aggressive form of disease, resulting in a 5-year survival rate of $47 \% .^{3}$ Transurethral resection of bladder tumor (TURBT), as the standard surgical treatment for NMIBC, contributes to some serious complications including obturator nerve reflex, bladder perforation, and bleeding. Given that TURBT is an "incise and scatter" procedure, and so issues such as tumor cell scattering, out-of-field recurrence, and incomplete resection plague the adequacy of TURBT. ${ }^{4}$ In addition, TURBT is not suitable for bladder cancer patients who take an oral anticoagulant because of an increased risk of bleeding. ${ }^{5}$ Therefore, efforts should be made to improve treatment strategies for NMIBC.
Correspondence: Guosheng Yang Department of Urology, Southern Medical University Affiliated Guangdong Second Provincial General Hospital, Southern Medical University, No 446 Xingang Middle Road, Guangzhou 5I03 17, People's Republic of China $\mathrm{Tel} / \mathrm{fax}+862089168668$

Email 2008yangguosheng@sina.com 
Laser therapy is safe and minimally invasive and provides an alternative option for treating NMIBC. Holmium (Ho) and thulium (Tm) laser techniques have been widely used in the treatment of NMIBC and achieve satisfactory outcomes. ${ }^{6}$ Potassium-titanyl-phosphate (KTP) green-light $532 \mathrm{~nm}$ laser is converted from 1,064 nm neodymium-doped yttrium aluminum garnet (Nd:YAG) laser with a KTP crystal. At present, mounting evidence has reported that 80 - or $120-\mathrm{W}$ KTP laser systems are popular and effective surgical methods for treating benign prostatic hyperplasia (BPH), especially for patients with cardiovascular diseases taking an oral anticoagulant. ${ }^{7,8}$ Recently, $\mathrm{He}$ et $\mathrm{al}^{9}$ initially reported that the $30-\mathrm{W}$ front-firing green-light laser en bloc enucleation technique is effective and safe for treating NMIBC. However, clinical evidence of green-light laser en bloc resection is still limited. Whether high-power green-light laser is suitable for the treatment of NMBIC remains unknown.

In the current study, we investigated the safety and efficacy of a 120-W front-firing green-light laser en bloc enucleation technique for treating patients with NMIBC.

\section{Patients and methods}

The Institutional Review Board of the Guangdong Second Provincial General Hospital approved this study (approval number: 2015-KYLL-062). The methods were performed in accordance with approved ethical guidelines. Informed consent was obtained from all eligible patients. From March 2014 to November 2015, NMIBC patients treated with either photoselective vapo-enucleation of bladder tumor (PVEBT) or TURBT performed by the same surgeon were retrospectively analyzed. Patients in the PVEBT group received En bloc resection. Surgical outcomes were compared between the 2 groups. The inclusion criteria were as follows: NMIBC with a diameter $\leq 3 \mathrm{~cm}$, tumor number $\leq 3$, and patients with at follow-up time of at least 12 months or until death. Exclusion criteria were presence of multiple or recurrent NMIBC, locally advanced BC (cT2 or higher) at CT examination, and/or distant metastases. A total of 64 patients were enrolled in the final analysis. Preoperative evaluation included clinical history, physical examination, as well as some basic condition of the patients.

\section{Operation procedure}

The patients were under combined spinal epidural anesthesia in a lithotomy position with continuous irrigation by physiologic saline (sodium chloride $0.9 \%$ ). The 120 -W front-firing green-light surgical laser system (Laserscope, Guangdong, People's Republic of China) was used for laser surgery. At the beginning of the procedure, we carefully examined the bladder and determined the tumor size, number, shape, and location. Then, a circular incision around the tumor base with a safety distance of $0.5-1.0 \mathrm{~cm}$ was used to label the planned removal area. The apparent vessels around the tumor base were coagulated, and then we deepened the prelabeled incision to the detrusor muscle layer and lifted the tumor by the laser vapor resection combined with the blunt dissection of the laser fiber tip. Bleeding spots were punctually coagulated (Figure 1). The tumor was removed by the laser vapor resection combined with the blunt dissection of the laser fiber tip. For the TURBT group, the tumor was resected piece by piece through a wire loop until the superficial muscular layer was exposed, and then the tumor was endoscopically extracted with an Ellik evacuator. Intravesical instillation chemotherapy with mitomycin $(40 \mathrm{mg} / 50 \mathrm{~mL})$ was performed within 24 hours after the operation for both groups. The catheter was removed if there was no gross hematuria. Intravesical instillation chemotherapy was performed weekly during the first 8 weeks after the operation. Twelve-month follow-up data were collected. Patients with hematuria or significant urinary symptoms required a referral.

\section{Cost analysis}

Costs were calculated from the hospital database and included operation and surgical instruments charges, examination charges, medicine charges, nursing charges, and total charges. The costs are expressed in US dollars (\$).

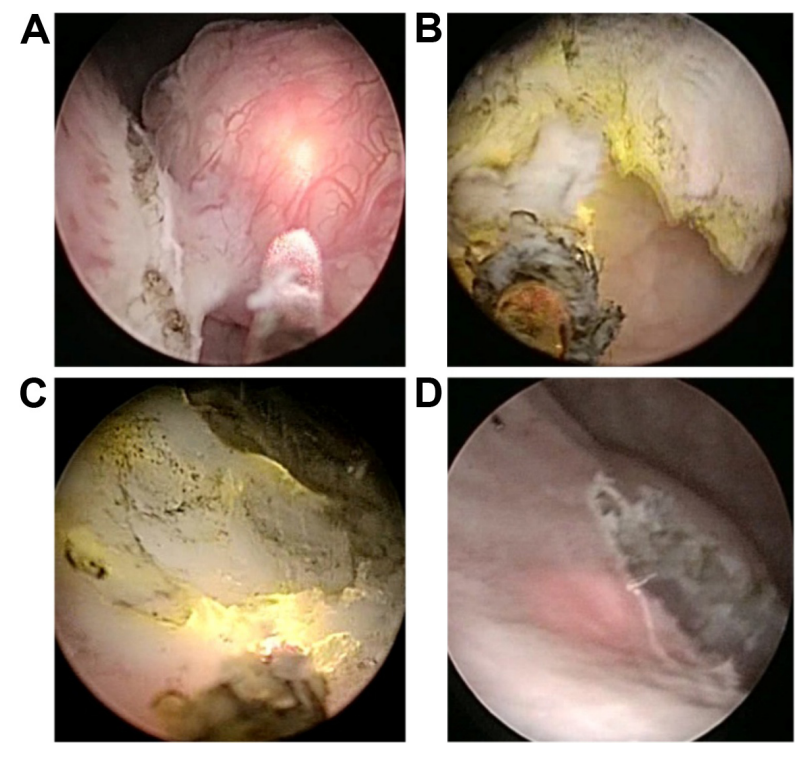

Figure I I20-W front-firing green-light laser en bloc enucleation technique. Notes: (A) A linear incision into the bladder wall around the lesion. (B) Removal of the tumor by the laser vapor resection combined with the blunt dissection of the laser fiber tip. (C) Surgical wound hemostasis. (D) Tumor base and surrounding tissue shown after tumor resection. 


\section{Statistical analysis}

SPSS 18.0 software (SPSS, Inc., Chicago, IL, USA) was used for statistical analyses. Qualitative variables were compared using the $\chi^{2}$ test with Fisher's correction when necessary. Student's $t$-test was used for normally distributed quantitative variables. Nonnormal continuous variables were compared using the Wilcoxon test. A $P$-value $<0.05$ was considered significant.

\section{Results}

\section{Patient characteristics}

The basic characteristics of patients are listed in Table 1 . A total of 64 patients with NMIBC were included. The PVEBT group comprised 34 patients. Thirty patients were enrolled in the TURBT group. No statistically significant difference was found between the two groups in terms of age $(P=0.217)$, gender $(P=0.483)$, hypertension $(P=0.385)$, hemoglobin decrease $(P=0.320)$, tumor size $(0.6334)$, location (0.3737), and diabetes $(P=0.363)$.

\section{Preoperative outcomes}

The preoperative outcomes are summarized in Table 1. There was no significant difference in rinsing time $(P=0.292)$ and indwelling catheter time $(P=0.080)$ between the two groups.

Table I Baseline characteristics and perioperative outcomes

\begin{tabular}{|c|c|c|c|}
\hline \multirow[t]{2}{*}{ Groups } & \multirow{2}{*}{$\frac{\text { PVEBT }}{\mathbf{N}=34}$} & \multirow{2}{*}{$\frac{\text { TURBT }}{\mathbf{N}=\mathbf{3 0}}$} & \multirow[t]{2}{*}{$P$-value } \\
\hline & & & \\
\hline \multicolumn{4}{|l|}{ Baseline characteristics } \\
\hline Age (year) & $59.41 \pm 12.99$ & $63.13 \pm 10.55$ & $0.2170^{\mathrm{a}}$ \\
\hline Gender (\%) & & & $0.483 \mathrm{I}^{\mathrm{b}}$ \\
\hline Male & $28(82.35)$ & $27(90.00)$ & \\
\hline Female & $6(17.65)$ & $3(10.00)$ & \\
\hline Diabetes (\%) & $5(14.70)$ & $3(10.00)$ & $0.3633^{b}$ \\
\hline Hypertension (\%) & $10(29.41)$ & $6(20.00)$ & $0.3855^{c}$ \\
\hline Tumor size $(\mathrm{cm})$ & $1.65(1.20-2.50)$ & $1.50(1.20-2.00)$ & $0.6334^{d}$ \\
\hline Location $(\%)$ & & & $0.3737^{\mathrm{b}}$ \\
\hline Trigone area & $13(38.24)$ & $7(23.33)$ & \\
\hline Anterior wall & I (2.94) & 0 & \\
\hline Posterior wall & $2(5.88)$ & I (3.33) & \\
\hline $\begin{array}{l}\text { Lateral bladder wall } \\
\text { (left/right) }\end{array}$ & 18 (52.94) & $22(73.33)$ & \\
\hline \multicolumn{4}{|c|}{ Perioperative outcomes } \\
\hline Operation time (min) & $35(30-45)$ & $30(20-40)$ & $0.0082^{d}$ \\
\hline Hemoglobin decrease & $5(3-9)$ & $7.5(3-10)$ & $0.3208^{d}$ \\
\hline Rinsing time & $19(16-23)$ & $21(17-27)$ & $0.2928^{d}$ \\
\hline Obturator nerve reflex & 0 & 10 & $0.0000^{\mathrm{b}}$ \\
\hline Indwelling catheter & $3(3-4)$ & $3.5(3-4)$ & $0.0808^{d}$ \\
\hline Hospitalization stay (day) & $5(4-6)$ & $5(5-6)$ & $0.0438^{d}$ \\
\hline
\end{tabular}

Notes: $P<0.05$ was considered statistically significant. ${ }^{2} T$ wo-sample independent $t$-test; ' ${ }^{\circ}$ Fisher's exact test; ${ }^{\circ} \chi^{2}$ test; ${ }^{d} W$ ilcoxon rank sum test.

Abbreviations: PVEBT, photoselective vapo-enucleation of the bladder tumor: TURBT, transurethral resection of the bladder tumor.
Table 2 The pathologic results of surgical staging

\begin{tabular}{|c|c|c|c|}
\hline \multirow{2}{*}{$\begin{array}{l}\text { Pathologic } \\
\text { diagnosis }\end{array}$} & \multirow{2}{*}{$\begin{array}{l}\text { PVEBT group } \\
\mathrm{N}=34\end{array}$} & \multirow{2}{*}{$\begin{array}{l}\text { TURBT group } \\
\mathbf{N}=30\end{array}$} & \multirow[t]{2}{*}{$P$-value } \\
\hline & & & \\
\hline PUNLMP (\%) & & & 0.5957 \\
\hline Papilloma & $6(17.65)$ & $6(20.00)$ & \\
\hline Low malignant & 7 (20.59) & $4(13.33)$ & \\
\hline potential & & & \\
\hline Low grade & $19(55.88)$ & $20(66.67)$ & \\
\hline High grade & $2(5.88)$ & 0 & \\
\hline T stage (\%) & & & 0.8699 \\
\hline $\mathrm{Ta}$ & $14(4|| 8)$. & $13(43.33)$ & \\
\hline TI & $16(47.06)$ & $15(50.00)$ & \\
\hline $\mathrm{T} 2 \mathrm{a}$ & $4(11.76)$ & $2(6.67)$ & \\
\hline Muscle miss rate (\%) & I (2.94) & $6(20.00)$ & 0.0444 \\
\hline
\end{tabular}

Abbreviations: PUNLMP, papillary urothelial neoplasms of low malignant potential; PVEBT, photoselective vapo-enucleation of the bladder tumor; TURBT, transurethral resection of the bladder tumor.

Compared with the TURBT group, patients treated with PVEBT had a shorter hospitalization stay $(P=0.044)$ and a shorter operation time $(P=0.008)$.

\section{Pathological results}

The pathological results of surgical staging are shown in Table 2. The PVEBT and TURBT groups did not differ with respect to pathologic type (papilloma, low malignant potential, low grade, high grade, $P=0.596)$ and T stage $(P=0.870)$. The muscle miss rate was significantly less in the PVEBT group than in the TURBT group $(P=0.044)$.

\section{Cost-effectiveness analysis}

As shown in Table 3, the operation and surgical instruments costs of the PVEBT group were significantly higher than those of the TURBT group $(P=0.000)$. In contrast, the costs for medication, examination, nursing, and treatment in the PVEBT group were lower than that in the TURBT group (all $P<0.01$ ). The total costs were similar between the two groups $(P=0.359)$.

Table 3 Costs comparisons between PVEBT and TURBT

\begin{tabular}{|c|c|c|c|}
\hline \multirow[t]{2}{*}{ Groups } & \multirow{2}{*}{$\frac{\text { PVEBT }}{\mathbf{N}=34}$} & \multirow{2}{*}{$\frac{\text { TURBT }}{\mathbf{N}=\mathbf{3 0}}$} & \multirow[t]{2}{*}{$P$-value } \\
\hline & & & \\
\hline Total costs & $2,854.87$ & $2,867.84$ & 0.359 \\
\hline $\begin{array}{l}\text { Operation and surgical } \\
\text { instruments }\end{array}$ & 865.72 & 675.83 & 0.000 \\
\hline Medication & 604.53 & 677.50 & 0.000 \\
\hline Examination & 443.33 & 481.55 & 0.001 \\
\hline Nursing and treatment & 729.74 & 805.71 & 0.000 \\
\hline Other & 211.55 & 227.25 & 0.059 \\
\hline
\end{tabular}

Abbreviations: PVEBT, photoselective vapo-enucleation of the bladder tumor; TURBT, transurethral resection of the bladder tumor. 


\section{Survival}

Postoperative prognoses are listed in Table 4. After at least 12-month follow-up, there were 3 recurrences in the PVEBT group, whereas 10 recurrences occurred in the TURBT group. The rate of 1-year recurrence was significantly lower in the PVEBT group than in the TURBT group $(P=0.015)$. There were 0 and 5 cases of progression of tumor grade found in the PVEBT and TURBT groups, respectively. The rate of 1-year tumor grade progression in the PVEBT group was much lower than in the TURBT group $(P=0.019)$. No significant difference was found between the two groups in terms of the number of recurrences at the original surgical site $(P=0.2158)$. The pathological diagnosis of the cases that recurred or progressed are listed in Table S1.

\section{Discussion}

This was a comparative study analyzing data on patients with NMIBC treated by PVEBT or TURBT. Recently, He et $\mathrm{al}^{9}$ first reported that the $30-\mathrm{W}$ front-firing green-light laser en bloc enucleation technique was effective and safe for treating NMIBC. However, whether high-power greenlight laser is suitable for the treatment of NMBIC remains unknown. Our results showed that patients who underwent PVEBT surgery (120-W front-firing green-light laser) had shorter operation time, lower muscle miss rate, and obturator nerve reflex rate, compared with TURBT. Notably, the rates of recurrence and tumor grade progression were significantly lower in the PVEBT group than in the TURBT group during the 12-month follow-up. The total hospital costs were similar between the two groups.

TURBT is one of the most commonly performed surgeries by practicing urologists. Multiple established guidelines recommend TURBT as the cornerstone of treatment for the initial diagnosis, staging, and treatment of NMIBC..$^{10,11}$ Although bladder tumors can be efficiently removed

Table 4 Recurrence and progression in PVEBT and TURBT groups

\begin{tabular}{|c|c|c|c|}
\hline \multirow[t]{2}{*}{ Groups } & \multirow{2}{*}{$\frac{\text { PVEBT group }}{\mathbf{N}=34}$} & \multirow{2}{*}{$\frac{\text { TURBT group }}{\mathbf{N}=30}$} & \multirow[t]{2}{*}{$P$-value } \\
\hline & & & \\
\hline Number of recurrence (\%) & $3(8.82)$ & $10(33.33)$ & $0.0150^{\mathrm{a}}$ \\
\hline $\begin{array}{l}\text { Number of progression of } \\
\text { tumor grade (\%) }\end{array}$ & 0 & $5(16.67)$ & $0.0187^{b}$ \\
\hline $\begin{array}{l}\text { Number of progression of } \\
\text { T stage (\%) }\end{array}$ & 0 & 0 & - \\
\hline $\begin{array}{l}\text { Number of recurrence at } \\
\text { the original surgical site (\%) }\end{array}$ & 0 & $2(6.67)$ & $0.2158^{\mathrm{b}}$ \\
\hline
\end{tabular}

Notes: ${ }^{ } \chi^{2}$ test; ' ${ }^{\circ}$ isher's exact test.

Abbreviations: PVEBT, photoselective vapo-enucleation of the bladder tumor; TURBT, transurethral resection of the bladder tumor. with TURBT, it has some potential risks. The common complications during surgery include severe bleeding, tumor cell implantation, and obturator nerve reflex resulting in bladder wall tears or perforation. ${ }^{12}$ Conventional TURBT leads to the scatter of malignant cells, which seems to ignore the principle of oncological surgery. A piecemeal resection of the tumor contributes to the high incidence of recurrence after initial TURBT. In addition, thermal damage causes difficulty in accurate pathological evaluation in fragmented tumor tissues after surgery. ${ }^{13}$ To overcome the shortcomings listed above, many surgical techniques have been developed. Laser ablation/resection techniques provide alternative options for treating NMIBC.

Currently, laser techniques are popularly used in urologic disease, such as BPH, bladder tumors, stricture, and urolithiasis, etc. ${ }^{14}$ Compared with TURBT, laser procedures have many advantages. The techniques are more precise and accurate. In addition, laser procedures are less invasive and result in less bleeding, swelling, and scarring. At present, the most commonly used lasers in clinical practice are Ho, YAG, Tm, YAG laser, KTP, and YAG laser, which is also referred to as green-light laser. ${ }^{14}$ Laser techniques were primarily applied to treat BPH for ablating prostatic tissue with minimal hemorrhaging. It has not been recommended for the treatment of bladder cancer because the approach gives rise to inadequate or no tissue for pathological evaluation. With the advancement of laser techniques, Ho laser was first reported for en bloc-resected bladder tumors in 2001, and then a Tm laser was used to treat bladder malignancy in $2008 .{ }^{15}$ Studies have suggested that Ho and Tm laser techniques are preferable alternatives for the management of NMIBC compared with TURBT, with fewer complications. ${ }^{16,17}$ Currently, mounting evidence has reported that $80-\mathrm{W}$ and $120-\mathrm{W}$ highperformance system KTP lasers are safe and feasible for men with BPH. ${ }^{18}$ However, clinical data on the application of the green-light laser in NMIBC are still rather limited. Tao et al have recently reported that the 120-W HPS KTP laser was a reliable therapeutic alternative for NMIBC and was superior to TURBT. ${ }^{19} \mathrm{He}$ et $\mathrm{al}{ }^{9}$ performed en bloc resection of NMIBC using a modeled $30-\mathrm{W}$ front-firing green-light laser, and their initial results were encouraging. Here, we evaluated the efficacy of "high-power" 120-W front-firing green-light laser for en bloc enucleation of NMIBC. Our findings indicated that the surgery times and hospitalization stays were shorter and the obturator nerve reflex rate was lower in the PVEBT group compared to the TURBT group. Several plausible hypotheses may explain the correlations. The green-light laser resects tumors in a noncontact way. Specifically, laser 
vaporization can seal off the blood vessels and lymph vessels around the tumor. The green-light laser possesses the property of excellent homeostasis, providing a bloodless operation field. Furthermore, postoperative bleeding is responsible for delayed recovery and leads to longer hospitalization stays. ${ }^{20}$ The control of local recurrence remains a great challenge to urologists for treating NMIBC. The KTP laser en bloc resects neoplasms in a deeper and wider range without touching the tumor tissue, which minimizes the risk for local recurrence. The results of our study have shown that 120-W front-firing green-light laser statistically significantly reduced the 1-year recurrence rate of NMIBC compared to TURBT.

Certain limitations existed in the present study. The major disadvantage of this analysis was its retrospective nature. The possibility of selection bias cannot be ruled out. In addition, another limitation of the study was the short follow-up period. However, considering that PVEBT is a new surgical approach and that the study provided the initial experience, 12-month follow-up seems acceptable. Larger prospective studies with a long follow-up period are necessary to confirm these findings.

\section{Conclusion}

In conclusion, our results suggested that PVEBT is superior to TURBT in terms of shorter operation times, a lower muscle miss rate, fewer complications, and a lower 1-year recurrence-free rate. PVEBT is a safe and effective treatment for NMIBC. Further validation of these results from large prospective trials with long-term follow-up is required.

\section{Acknowledgment}

This work was funded by Science and Technology Planning Project of Guangdong Province (No 2014A020212680).

\section{Disclosure}

The authors report no conflicts of interest in this work.

\section{References}

1. Miller KD, Siegel RL, Lin CC, et al. Cancer treatment and survivorship statistics, 2016. CA Cancer J Clin. 2016;66(4):271-289.

2. Burger M, Catto JW, Dalbagni G, et al. Epidemiology and risk factors of urothelial bladder cancer. Eur Urol. 2013;63(2):234-241.
3. Babjuk M, Bohle A, Burger M, et al. EAU Guidelines on non-muscleinvasive urothelial carcinoma of the bladder: update 2016. Eur Urol. 2017;71(3):447-461.

4. Mariappan P, Zachou A, Grigor KM. Detrusor muscle in the first, apparently complete transurethral resection of bladder tumour specimen is a surrogate marker of resection quality, predicts risk of early recurrence, and is dependent on operator experience. Eur Urol. 2010; 57(5):843-849.

5. De Nunzio C, Franco G, Cindolo L, et al. Transuretral resection of the bladder (TURB): analysis of complications using a modified Clavien system in an Italian real life cohort. Eur J Surg Oncol. 2014; 40(1):90-95.

6. Herrmann TR, Liatsikos EN, Nagele U, Traxer O, Merseburger AS. EAU guidelines on laser technologies. Eur Urol. 2012;61(4):783-795.

7. Bachmann A, Muir GH, Collins EJ, et al. 180-W XPS GreenLight laser therapy for benign prostate hyperplasia: early safety, efficacy, and perioperative outcome after 201 procedures. Eur Urol. 2012;61(3): 600-607.

8. Kuntz RM. Current role of lasers in the treatment of benign prostatic hyperplasia (BPH). Eur Urol. 2006;49(6):961-969.

9. He D, Fan J, Wu K, et al. Novel green-light KTP laser en bloc enucleation for nonmuscle-invasive bladder cancer: technique and initial clinical experience. J Endourol. 2014;28(8):975-979.

10. Babjuk M, Burger M, Zigeuner R, et al. EAU guidelines on non-muscleinvasive urothelial carcinoma of the bladder: update 2013. Eur Urol. 2013;64(4):639-653.

11. Power NE, Izawa J. Comparison of guidelines on non-muscle invasive bladder cancer (EAU, CUA, AUA, NCCN, NICE). Bladder Cancer. 2016;2(1):27-36.

12. Jancke G, Rosell J, Jahnson S. Impact of surgical experience on recurrence and progression after transurethral resection of bladder tumour in non-muscle-invasive bladder cancer. Scand J Urol. 2014; 48(3):276-283.

13. Gregg JR, McCormick B, Wang L, et al. Short term complications from transurethral resection of bladder tumor. Can J Urol. 2016;23(2): 8198-8203.

14. Zarrabi A, Gross AJ. The evolution of lasers in urology. Ther Adv Urol. 2011;3(2):81-89.

15. Saito S. Transurethral en bloc resection of bladder tumors. $J$ Urol. 2001;166(6):2148-2150.

16. Zhong C, Guo S, Tang Y, Xia S. Clinical observation on 2 micron laser for non-muscle-invasive bladder tumor treatment: single-center experience. World J Urol. 2010;28(2):157-161.

17. Zhu Y, Jiang X, Zhang J, Chen W, Shi B, Xu Z. Safety and efficacy of holmium laser resection for primary nonmuscle-invasive bladder cancer versus transurethral electroresection: single-center experience. Urology. 2008;72(3):608-612.

18. Tao W, Xue B, Zang Y, et al. The application of 120-W high-performance system GreenLight laser vaporization of the prostate in high-risk patients. Lasers Med Sci. 2013;28(4):1151-1157.

19. Tao W, Yang D, Shan Y, et al. Safety and efficacy of $120 \mathrm{~W}$ high performance system greenlight laser vaporization for non-muscle-invasive bladder cancer. J Xray Sci Technol. 2013;21(2):309-316.

20. Xu Y, Guan W, Chen W, et al. Comparing the treatment outcomes of potassium-titanyl-phosphate laser vaporization and transurethral electroresection for primary nonmuscle-invasive bladder cancer: A prospective, randomized study. Lasers Surg Med. 2015;47(4):306-311. 


\section{Supplementary material}

Table SI The pathological diagnosis of the cases that recurred or progressed

\begin{tabular}{llll}
\hline Case & Treatment & Pathological diagnosis & R or/and $\mathbf{P}$ \\
\hline I & PVEBT & Low-grade papillary urothelial carcinoma & $\mathrm{R}$ \\
2 & PVEBT & Low-grade urothelial carcinoma & $\mathrm{R}$ \\
3 & PVEBT & Low-grade papillary urothelial carcinoma & $\mathrm{R}$ \\
4 & TRUBT & High-grade urothelial carcinoma with squamous differentiation & $\mathrm{R}$ \\
5 & TRUBT & Low degree of malignant potential urothelial carcinoma & $\mathrm{R}$ \\
6 & TRUBT & Low-grade urothelial carcinoma & $\mathrm{R}$ \\
7 & TRUBT & Low-grade urothelial carcinoma & $\mathrm{R}$ and P \\
8 & TRUBT & Low-grade papillary urothelial carcinoma & $\mathrm{R}$ \\
9 & TRUBT & Low-grade urothelial carcinoma & $\mathrm{R}$ and P \\
10 & TRUBT & Inverted urothelial papilloma & $\mathrm{R}$ and P \\
II & TRUBT & Low degree of malignant potential urothelial carcinoma & $\mathrm{R}$ and P \\
I2 & TRUBT & Urothelial carcinoma & $\mathrm{R}$ \\
I3 & TRUBT & Low degree of malignant potential papillary urethral carcinoma & $\mathrm{R}$ and P \\
\hline
\end{tabular}

Abbreviations: $\mathrm{P}$, progression; PVEBT, photoselective vapo-enucleation of the bladder tumor; $\mathrm{R}$, resection; TURBT, transurethral resection of the bladder tumor.

\section{Publish your work in this journal}

Therapeutics and Clinical Risk Management is an international, peerreviewed journal of clinical therapeutics and risk management, focusing on concise rapid reporting of clinical studies in all therapeutic areas, outcomes, safety, and programs for the effective, safe, and sustained use of medicines. This journal is indexed on PubMed Central, CAS,
EMBase, Scopus and the Elsevier Bibliographic databases. The manuscript management system is completely online and includes a very quick and fair peer-review system, which is all easy to use. Visit http://www.dovepress.com/testimonials.php to read real quotes from published authors. 